\title{
Drowning in swimming pools: clinical features and safety recommendations based on a study of descriptive records by emergency medical services attending to 995 calls
}

\author{
Joanna Shi-En $\underline{\mathrm{Chan}^{1}}$, MBвs, MCEM, Marie Xin $\mathrm{Ru} \underline{\mathrm{Ng}^{2}}$, BSc, MPH, Yih Yng $\underline{\mathrm{Ng}^{2}}$, MBBs, MPH
}

\begin{abstract}
INTRODUCTION This study was a descriptive analysis of national ambulance case records and aimed to make practical safety recommendations in order to reduce the incidence of drowning in swimming pools.

METHODS A search was performed of a national database of descriptive summaries by first-responder paramedics of all 995 calls made to the Singapore Civil Defence Force between 1 January 2012 and 31 December 2014. We included all cases of submersion in both public and private pools for which emergency medical services were activated.

RESULTS The highest proportion of drowning cases occurred in the age group of $0-9$ years. Males accounted for $57.0 \%$ $(61 / 107)$ of cases. Bystander cardiopulmonary resuscitation (CPR) was performed in 91.3\% (21/23) and 68.6\% (48/70) of cases of cardiac/respiratory arrest from drowning in public and private pools, respectively; the rate of bystander CPR was higher when a lifeguard was present $(88.5 \%, 23 / 26$ vs. $68.7 \%, 46 / 67)$. The majority $(72.0 \%, 77 / 107)$ of drowning incidents occurred in private pools, most of which had no lifeguards present.

CONCLUSION To our knowledge, this study was the first in Singapore to examine data from emergency medical services. Since the majority of incidents occurred in private pools without lifeguards, it is recommended that a lifeguard be present at every pool. For pools that are too small to justify mandatory lifeguard presence, safety measures, such as guidelines for pool design and pool fencing with latched gates, may be considered. As strict enforcement may not be possible, public education and parental vigilance remain vital.
\end{abstract}

Keywords: accident prevention, drowning, prehospital emergency care, public health, swimming pools

\section{INTRODUCTION}

There were an average of 73 cases per year of death by drowning in Singapore between 1965 and 2005. ${ }^{(1)}$ Drowning rates ranged from 0.88 to 1.72 per 100,000 from 1992 to 2001, (2) and decreased to 0.39 and 0.18 per 100,000 in 2012 and 2013, respectively. ${ }^{(3)}$ This is comparable to the drowning rates in other high-income countries such as Australia, Sweden and the United Kingdom. ${ }^{(4)}$ Nevertheless, drowning prevention continues to be a relevant concern, as a large number of people, mostly children, go swimming in Singapore's equatorial climate. Based on reports by the Singapore Life Saving Society (SLSS), 117 cases of death by drowning were heard in the Coroner's Court during the period 2012-2014. ${ }^{(5)}$ Of these, 19 took place in swimming pools. (Note: the annual reports of the SLSS are not primary sources of data. Data derived from the Coroner's Court is based on cases where drowning was mentioned or suspected for the coroner's inquiries with pending verdicts and pronounced verdicts, and do not tally exactly with figures from the Registry of Births and Deaths, which exclude instances of assault, self-harm and death from other causes in cases classified as accidental drowning.)

In 2014, there was an attendance of 6,870,183 at swimming pools operated by the Singapore Sports Council (SSC). ${ }^{(6)}$ Swimming was the second most popular sport in the 2011 National Sports Participation Survey and the top sport that children aged $\leq 12$ years engaged in outside of school. ${ }^{(7)}$ The need to review drowning safety in swimming pools is especially pertinent given the increase in the number of swimming pools in the country. The National Environment Agency reported that there were 1,889 swimming pool licences in force in 2012, an increase from 1,772 in 2011. ${ }^{(8)}$ This figure applies to pools operated by the SSC, school campuses, hotels and condominiums, but does not include landed home pools, which do not require a licence. ${ }^{(9)}$ The number of such pools in Singapore is unknown but likely to be on the rise.

The aim of the present study was to conduct a descriptive analysis of the national ambulance case records, so as to make practical safety recommendations to reduce the danger of drowning incidents in swimming pools. We postulated that a higher rate of bystander cardiopulmonary resuscitation (CPR) might occur in the presence of a lifeguard. A literature review showed that no similar study has previously been published in Singapore.

\section{METHODS}

A categorical and free text search was performed of a national electronic database of descriptive summaries by first-responder paramedics of all 995 calls made to the Singapore Civil Defence Force (SCDF) between 1 January 2012 and 31 December 2014. 
The purpose was to identify all likely cases of drowning in both public and private pools for which emergency medical services were activated. These summaries had originally been written freehand on paper by paramedics who had conveyed patients to hospital or pronounced them dead at the scene. The summaries were then transcribed by the paramedics and archived in an electronic database. Institutional review board approval and waiver of consent were obtained for this study.

We included all cases of submersion in swimming pools for which the SCDF was called during the study period. We understood drowning as referring to all cases of submersion injury regardless of whether death occurred, in accordance with the uniform reporting guidelines proposed by Idris et al. ${ }^{(10)}$ Cases in which the patient lost consciousness in the water due to another cause but was at risk of submersion injury were also included. For the purpose of this study, swimming pools run by the SSC and those located in public educational institutions were referred to as 'public pools', and swimming pools in private housing (condominiums and landed homes) and hotel or leisure facilities were referred to as 'private pools'. We excluded all cases of submersion injury that did not take place at a swimming pool. After a free text review, we further excluded cases that involved illness without submersion (e.g. partial seizure while standing in water), or cases in which the victim was pronounced dead at the scene after a presumably long period of submersion and it was unclear if the death was a result of submersion or due to foul play.

Data gathered for each incident included information on age, gender, lifeguard presence, performance of bystander CPR, use of an automated external defibrillator (AED) and clinical features. A lifeguard was taken to be present if the paramedic's summary explicitly stated the presence of a lifeguard or swimming instructor. The mention of 'staff' or 'security personnel' performing CPR was not regarded as lifeguard presence.

The victims were identified as having a cardiac arrest if they were described as pulseless. If they were described as apnoeic or unconscious after submersion, with or without documented cyanosis, they were deemed to have suffered a respiratory arrest. Additional features of the case (such as whether the submersion was witnessed) were noted if they had been documented by paramedics.

\section{RESULTS}

A total of 107 drowning cases were identified in our search, with $77(72.0 \%)$ occurring in private pools (Fig. 1). Drowning most frequently occurred in the age group of $0-9$ years $(42.1 \%$, 45/107). A further $26.2 \%$ (28/107) of drowning cases involved adults aged $\geq 40$ years (Table I). Seven cases involved children aged 2-6 years who were not swimming but slipped or were pushed into the pool. Of the seven cases, six occurred in private pools. In one case, a toddler had wandered off from a poolside family function and fallen into the pool. Another case involved an infant left unattended for 30 seconds in a home baby pool.

The clinical features of the cases are described in Table II. There were $24(22.4 \%)$ cases of cardiac arrest without return of spontaneous circulation (ROSC). In two of these cases, it was
Table I. Age and gender of persons who drowned $(n=107)$.

\begin{tabular}{lccc}
\hline Age $(\mathbf{y r})$ & \multicolumn{3}{c}{ No. (\%) } \\
\cline { 2 - 4 } & Male & Female & Total \\
\hline $0-9$ & $26(24.3)$ & $19(17.8)$ & $45(42.1)$ \\
$10-19$ & $10(9.3)$ & $8(7.5)$ & $18(16.8)$ \\
$20-29$ & $4(3.7)$ & $5(4.7)$ & $9(8.4)$ \\
$30-39$ & $6(5.6)$ & $1(0.9)$ & $7(6.5)$ \\
$40-49$ & $3(2.8)$ & $7(6.5)$ & $10(9.3)$ \\
$50-59$ & $4(3.7)$ & $2(1.9)$ & $6(5.6)$ \\
$60-69$ & $3(2.8)$ & $2(1.9)$ & $5(4.7)$ \\
$\geq 70$ & $5(4.7)$ & $2(1.9)$ & $7(6.5)$ \\
Total & $61(57.0)$ & $46(43.0)$ & $107(100.0)$ \\
\hline
\end{tabular}

Table II. Clinical features of cases as described by paramedics in free text notes.

\begin{tabular}{lccc}
\hline Clinical feature & \multicolumn{3}{c}{ No. (\%) } \\
\cline { 2 - 4 } & $\begin{array}{c}\text { Regained } \\
\text { consciousness } \\
\text { at scene }\end{array}$ & $\begin{array}{c}\text { Did not regain } \\
\text { consciousness } \\
\text { at scene }\end{array}$ & Total \\
\hline $\begin{array}{l}\text { Cardiac arrest } \\
\text { without ROSC }\end{array}$ & - & $24(22.4)$ & $24(22.4)$ \\
$\begin{array}{l}\text { Cardiac arrest } \\
\text { with ROSC }\end{array}$ & $6(5.6)$ & $3(2.8)$ & $9(8.4)$ \\
$\begin{array}{l}\text { Respiratory } \\
\text { arrest* }\end{array}$ & $52(48.6)$ & & \\
$\begin{array}{l}\text { No loss of } \\
\text { consciousness } \\
\text { Loss of } \\
\begin{array}{l}\text { consciousness due } \\
\text { to other reasons } \\
\text { Total }\end{array}\end{array}$ & $11(10.3)$ & & $11(10.3)$ \\
\hline
\end{tabular}

*Respiratory arrest was defined as documentation of no breathing or loss of consciousness after submersion in the presence of a pulse. ROSC: return of spontaneous circulation

unclear whether there was a preceding medical event: one adult felt giddy and collapsed in the baby pool prior to submersion, while another, with bruises on the forehead, was pulled out after the unwitnessed event. Three victims lost consciousness due to reasons other than drowning: seizure in two adults aged 28 years and 38 years, and trauma in a ten-year-old child who jumped from height into the pool. Other clinical features were documented by the paramedics for 80 of the 107 cases, of which 50 (62.5\%) were reported to be unwitnessed at the moment of drowning and the victims were found unresponsive. In ten cases, the period in which the victim was unsupervised was documented as ranging from less than 30 seconds to 45 minutes.

The presence of a lifeguard was documented in 33 out of 107 cases (30.8\%; Fig. 2). Among these drowning incidents with lifeguard presence, the majority $(78.8 \%, 26 / 33)$ took place in public pools. Lifeguard involvement in rescue or resuscitation was noted in $26(86.7 \%)$ of the 30 cases of drowning in public pools, whereas lifeguard presence was not documented in $90.9 \%$ (70/77) cases of drowning in private pools. In public and private pools, respectively, there were 23 cases and 70 cases of cardiac or respiratory arrest from drowning. Bystander CPR was 


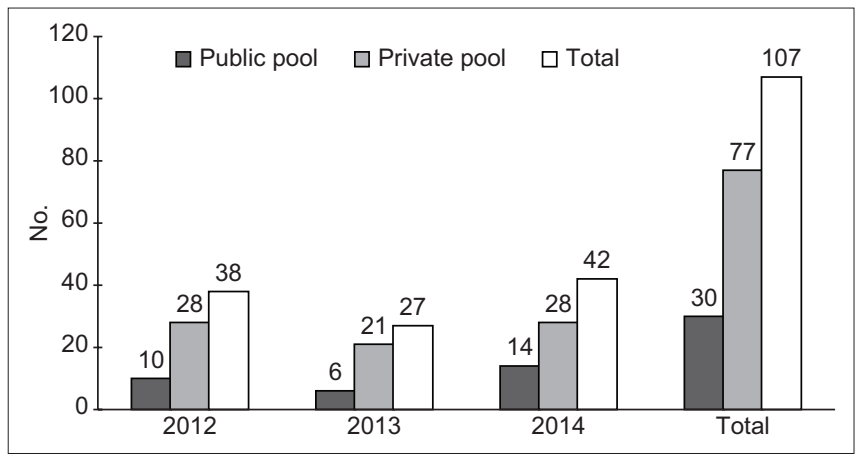

Fig. 1 Graph shows the incidence of drowning in pools between 2012 and 2014.

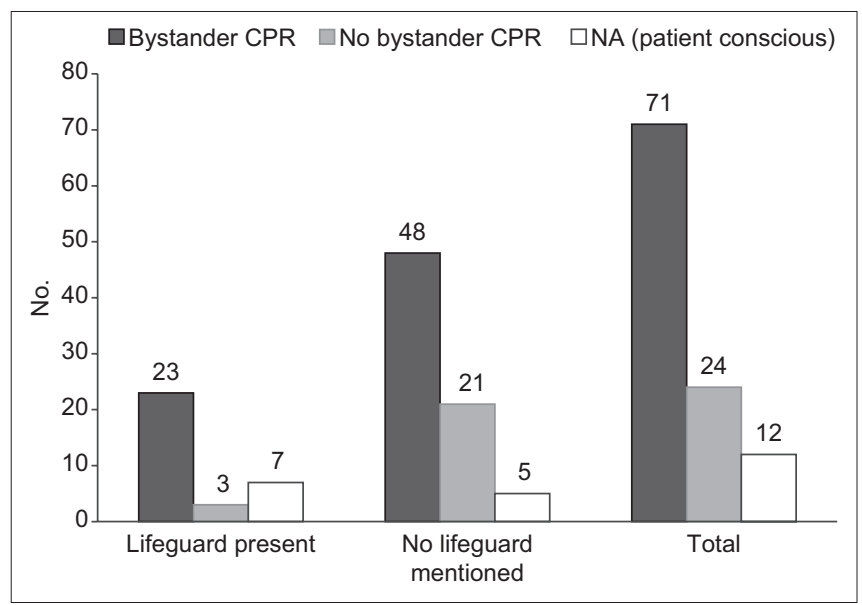

Fig. 3 Graph shows the prevalence of bystander cardiopulmonary resuscitation (CPR) in drowning cases with and without the presence of lifeguards. NA: not applicable

performed in $91.3 \%(\mathrm{n}=21)$ of the cases in public pools and in $68.6 \%(n=48)$ of the cases in private pools. In cases of cardiac or respiratory arrest from drowning, the overall rate of bystander CPR was $74.2 \%(69 / 93)$, and the rate of bystander CPR was $88.5 \%(23 / 26)$ in the presence of a lifeguard and $68.7 \%(46 / 67)$ in the absence of a lifeguard. In four cases, the patients regained consciousness with rescue breaths alone.

In cases where lifeguard presence was not documented, the patients were less likely to receive CPR (Fig. 3). No bystander CPR occurred in four cases of cardiac arrest without prehospital ROSC (Fig. 4). In two of these cases, the patients were pronounced dead at the scene. It was mentioned in seven cases that an AED was applied prior to the arrival of the ambulance. In six out of these seven cases, AED use was initiated by a lifeguard at a public pool. In the one remaining case, the AED was operated by a doctor at a private hotel pool. In four out of seven cases, the rhythm was shockable and one or more shocks were delivered prior to the arrival of an ambulance; one such patient experienced prehospital ROSC. In two cases where an AED was not used, shocks were delivered by paramedics after the arrival of the ambulance, but this did not result in prehospital ROSC.

In five cases, the drowning victims were reported to be nonswimmers, and in another, the victim was a former competitive swimmer. In two adult cases, alcohol use by the victims prior to drowning was documented by paramedics.

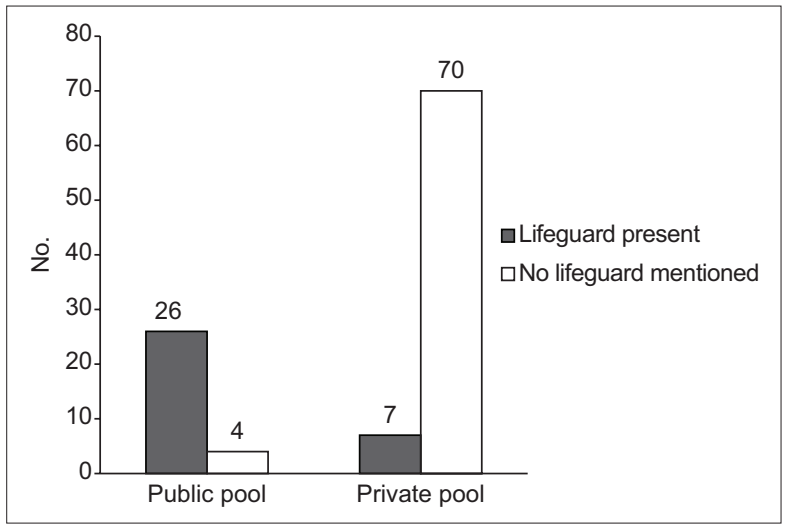

Fig. 2 Graph shows the recorded instances of lifeguard presence in drowning incidents occurring in public and private pools.

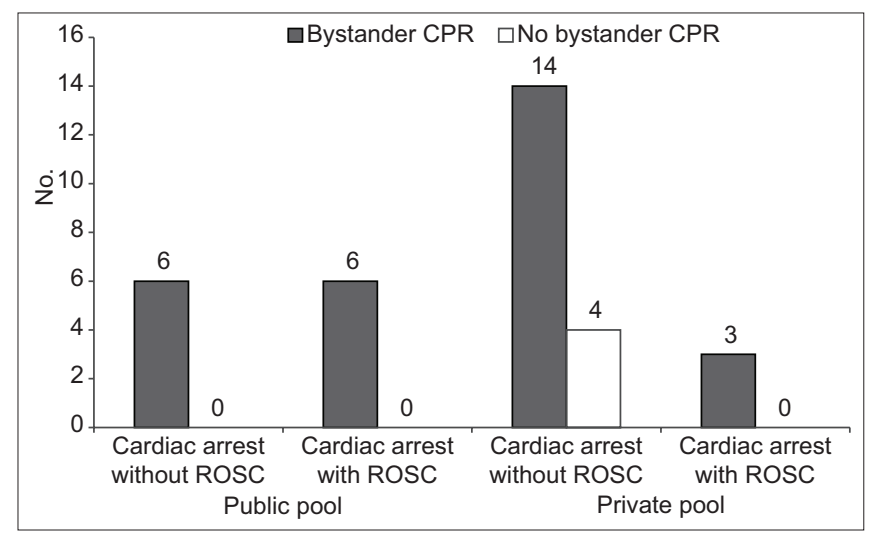

Fig. 4 Graph shows cardiac arrest prehospital outcomes and prevalence of bystander cardiopulmonary resuscitation (CPR). ROSC: return of spontaneous circulation

\section{DISCUSSION}

This is the first local study to examine data from emergency medical services. There were 19 swimming pool deaths by drowning heard in the Coroner's Court during our study period - four in public pools and 15 in private pools, ${ }^{(5)}$ although the SCDF was activated for 107 swimming pool drowning incidents during this period.

The present study confirmed our hypothesis that the rate of bystander CPR is higher in the presence of a lifeguard. It is worrisome that the majority (77 of 107 cases) of drowning incidents attended to by the SCDF occurred in private pools, most of which do not have lifeguards. A lifeguard was documented to be present in only $9.1 \%$ of drowning cases in private pools, as compared to $86.7 \%$ of cases in public pools. We postulate that lifeguard presence may improve response time to a drowning incident, shorten the time taken to initiate CPR and provide good quality CPR by trained personnel. In a paediatric study, Kyriacou et al reported that children who experienced drowning with a good outcome were 4.75 times (adjusted odds ratio) more likely to have experienced immediate resuscitation than children with poor outcomes (95\% confidence interval 3.44-6.06; $p=0.0001) .{ }^{(11)}$ Conversely, when CPR is delayed, of inferior quality or not initiated, there is a danger of poor clinical outcomes. In the present study, bystander CPR for cardiac or respiratory arrest from drowning was performed in a higher percentage of cases 
in public pools compared to private pools, which may be due to greater lifeguard presence in the former.

Interestingly, we found that even when no lifeguard was present, the incidence of bystander CPR in our study was more than three times higher than that of general bystander CPR previously reported in Singapore (15\%-23\%). ${ }^{(12)}$ We hypothesise that the reasons for this, while unclear, include the influence of media (e.g. television) on public perception of what to do in a drowning case and the high number of paediatric cases, in which distaste on the part of the public to perform mouth-tomouth resuscitation on a stranger may present less of a barrier. Nevertheless, patients with respiratory arrest were more likely to be conscious at the scene. We postulate that many of them who did not receive bystander CPR may have regained consciousness before CPR could be initiated.

Drowning incidents in private pools have recently come under the spotlight due to an apparent increase in childhood drowning incidents. Recent data from a study done at KK Women's and Children's Hospital, Singapore, showed that 69 out of 104 drowning incidents involving children between 2011 and 2015 took place in private pools. Of these, ten children died and two suffered irreversible brain injury. The hospital saw an average of 20 cases of drowning per year during the five-year period - an increase from a rate of 1-14 cases annually from 2005 to $2010 .^{(13)}$

Previous local studies have highlighted that private pools are likely to lack lifeguard supervision, despite lifeguard supervision having been associated with a reduction in drowning incidents. ${ }^{(2)}$ In an emergency department case series, 11 out of 17 drowning incidents occurred in swimming pools, and CPR was delayed or absent for two patients who drowned in private pools, one of whom suffered hypoxic brain injury. ${ }^{(14)}$ Also, according to the Childhood Injury Surveillance Project, 11 out of 20 swimming pool drowning incidents in children occurred in private pools during the period of 2002-2004. (15) In a separate local review of injuries in children attending the emergency department, most of the swimming pool near-drowning cases and one of the two drowning deaths involved private pools. ${ }^{(16)}$

In the present study, the age group of 0-9 years accounted for the highest proportion of drowning cases, which underscores the need for close parental supervision of children in this age group. About $20 \%$ of incidents in our study involved children under five years of age. Drowning fatalities in this age group are the highest worldwide and most often associated with swimming pools, ${ }^{(4)}$ although these children are also at risk from drowning at home. ${ }^{(4,13-16)} 26.2 \%$ of the cases in the present study involved victims aged 40 years and above, for whom comorbidities or unexpected medical events may have precipitated drowning and become a consideration in management. In two cases, the drowning was precipitated by seizures. In several adult cardiac arrest cases, it was unclear if there was a preceding medical event causing collapse while the patients were swimming. This is a useful reminder for first responders and clinicians involved in the care of drowning cases to look for precipitating medical events in all drowning cases.
Drowning prevention strategies worldwide can be classified into four broad categories: environmental modification; education and skills; legislation and standards; and immediate resuscitation. ${ }^{(2,4,15,17-19)}$ The World Health Organization and the American Academy of Pediatrics place the greatest emphasis on pool fencing, 'touch' supervision by a parent within arm's length for infants and toddlers, and the availability of CPRtrained personnel at the scene, in view of the short window of opportunity for rescue intervention..$^{(4,20)}$ While there have been no formal studies evaluating the effectiveness of lifeguards in the prevention of drowning, before-and-after data on rescue and resuscitation in the United States (US) suggested that supervision by lifeguards saves lives and lowers drowning rates. ${ }^{(21)}$ Besides rescue, lifeguards also play a role in surveillance and prevention, and a lifeguard trained in CPR can aid in immediate resuscitation measures at the scene.

In our local setting, the National Water Safety Council has previously urged lifeguard attendance at private pools. ${ }^{(22,23)}$ While there should ideally be a lifeguard in attendance at every pool, it may not be practical or cost-effective to provide a lifeguard in the setting of a pool in a landed home or a small shared facility with a low volume of swimmers. A logical first step may be to formally define the characteristics of a pool that requires a lifeguard, decide what constitutes proper lifeguard training and mandate lifeguard presence at such pools. In addition, in premises that lack lifeguards, a practical step would be to train security officers on the use of CPR and AED so that there are trained personnel to render immediate resuscitative assistance at the scene should a drowning incident occur.

It is currently not required by the law for operators of swimming pools to enforce lifeguard presence. However, in the case of BNM v. National University of Singapore [2014] SGHC 5, the Singapore High Court decided that owners or operators of at least standard Olympic-sized pools may owe a duty to users of the pool to provide properly trained lifeguards. ${ }^{(24)}$ Likewise, there is currently no legislation to enforce safety standards in pool design. The Singapore Standard (SS) 556: 2010 Code of Practice for the maintenance and design of aquatic facilities was launched in 2010 with the aim of minimising swimming injury and preventable drowning incidents. ${ }^{(25)}$ However, compliance with the SS 556 is not mandatory but dependent on the conscientiousness of industry players.

Other safety measures could be considered in pools that are too infrequently used to justify mandatory lifeguard presence. Medical professionals and building managers in Singapore have issued calls for legislation of pool design and pool fencing. ${ }^{(13-15)}$ While rigorous studies with high-level evidence are lacking, ${ }^{(26)}$ there is empiric evidence from the US, Australia and New Zealand of the protective effect of pool fencing against childhood drowning, particularly in the age group of $0-4$ years. ${ }^{(27-33)}$ Legislation for pool fencing has also been shown to decrease drowning deaths, ${ }^{(34-36)}$ although not to the extent that might be anticipated, likely due to inadequate enforcement. ${ }^{(37-39)}$ As landed home pools become more prevalent here, pool fencing to prevent unattended children from wandering into a home pool 
may become an increasingly relevant topic for discussion in the local context. ${ }^{(15,20)}$ As home pools are currently not subject to any safety standards or auditing process, a standard advice package highlighting drowning prevention measures could be provided to owners of home pools upon their creation, and these owners should be encouraged to learn CPR. Likewise, public education packages can also be given to buyers of inflatable pools and pool equipment.

The present study was not without limitations. Firstly, it was a simple retrospective database search. The cases in our review were found if they contained the relevant keywords or if data in the available categories (e.g. location) suggested a swimming pool drowning incident. Thus, we might have missed cases that could not be found based on free-text searches or categorical searches. As such, the incidence of drowning cases reported in this review may be an underestimation of the true number. Cases for which SCDF ambulances were not called were not represented in this review and we did not follow up on hospital outcomes. Lastly, there is no national standard for data collection to examine the causes of drowning, and the retrospective data record analysis has limitations in elucidating the causal factors that lead to drowning. Such requirements can be considered for future trauma database collection and data analysis to enable more systematic research on the causes of drowning.

In conclusion, public education remains vital in reducing drowning in swimming pools. Regardless of age or experience, even trained swimmers should be discouraged from swimming alone. Adults should refrain from going near any body of water if intoxicated or feeling unwell. Infants and young children who are not water-safe should be under continuous adult supervision when they are around swimming pools. ${ }^{(3,4,15,16)}$ Parental vigilance applies equally to children attending a poolside function and those with the intention to swim.

\section{ACKNOWLEDGEMENT}

We thank Mr Richard Tan Ming Kirk, President of the Singapore Life Saving Society, for providing the data on drowning cases from the Coroner's Court.

\section{REFERENCES}

1. Ministry of Social and Family Development, Republic of Singapore. Water Safety Task Force Final Report (2007). Available at: http://app.msf.gov.sg/portals/0/ files/watersafetytaskforcefinalreport.pdf. Accessed March 15, 2016.

2. Tan RM. The epidemiology and prevention of drowning in Singapore. Singapore Med J 2004; 45:324-9.

3. Registry of Births and Deaths, Immigration and Checkpoints Authority, Singapore. Report on Registration of Births and Deaths 2013. Available at: https:// www.ica.gov.sg/data/resources/docs/Media\%20Releases/SDB/Annual\%20 RBD\%20Report_2013.pdf. Accessed March 15, 2016.

4. Peden M, Oyegbite K, Ozanne-Smith J, et al. World Report on Child Injury Prevention. In: WHO Guidelines Approved by the Guidelines Review Committee. Geneva: World Health Organization, 2008.

5. Singapore Life Saving Society. Annual reports (2012-2014).

6. Department of Statistics, Ministry of Trade and Industry, Republic of Singapore. Yearbook of Statistics Singapore, 2015. Available at: http://www.singstat.gov. sg/docs/default-source/default-document-library/publications/publications_and_ papers/reference/yearbook_2015/yos2015.pdf. Accessed March 15, 2016.

7. Singapore Sports Council. National sports participation survey 2011 final report. Available at: https://www.sportsingapore.gov.sg/about-us/ /media/corporate/ files/about/publications/national\%20sports\%20participation $\% 20$ survey $\% 20$ 2011.pdf. Accessed March 15, 2016.
8. Tai J. Singapore Sports Council issues call for lifeguards as number of pools surges. The Straits Times 2013 Apr 21.

9. National Environment Agency, Singapore. Swimming pool licence. Available at: http://www.nea.gov.sg/services-forms/licences-permits-building-planclearances/others/swimming-pool-licence. Accessed March 15, 2016,

10. Idris AH, Berg RA, Bierens J, et al; American Heart Association; Maatschappij tot Redding van Drenkelingen; European Resuscitation Council; US Centers for Disease Control and Prevention; University of Florida; University of North Carolina; Australia and New Zealand Resuscitation Council; InterAmerican Heart Foundation; Heart and Stroke Foundation of Canada; Resuscitation Council of Southern Africa. Recommended guideline for uniform reporting of data from drowning: the "Utstein style". Resuscitation 2003; 59:45-57.

11. Kyriacou DN, Arcinue EL, Peek C, Kraus JF. Effect of immediate resuscitation on children with submersion injury. Pediatrics 1994; 94(2 Pt 1):137-42.

12. Leong BS. Bystander CPR and survival. Singapore Med J 2011; 52:573-5.

13. Lai L. Half of drownings involving kids happened in condo pools. The Straits Times 2016 Mar 11.

14. Goh SH, Low BY. Drowning and near-drowning--some lessons learnt. Ann Acad Med Singapore 1999; 28:183-8.

15. Tyebally A, Ang SY. Kids can't float: epidemiology of paediatric drowning and near-drowning in Singapore. Singapore Med J 2010; 51:429-33.

16. Ong ME, Ooi SB, Manning PG. A review of 2,517 childhood injuries seen in a Singapore emergency department in 1999--mechanisms and injury prevention suggestions. Singapore Med J 2003; 44:12-9.

17. Mott TF, Latimer KM. Prevention and treatment of drowning. Am Fam Physician 2016; 93:576-82.

18. Bernard SJ, Paulozzi LJ, Wallace DL; Centers for Disease Control and Prevention (CDC). Fatal injuries among children by race and ethnicity--United States, 1999-2002. MMWR Surveill Summ 2007; 56:1-16.

19. Orlowski JP, Szpilman D. Drowning. Rescue, resuscitation, and reanimation. Pediatr Clin North Am 2001; 48:627-46.

20. American Academy of Pediatrics Committee on Injury, Violence, and Poison Prevention. Prevention of drowning. Pediatrics 2010; 126:178-85.

21. Branche CM, Stewart S, eds. Lifeguard Effectiveness: A Report of the Working Group. Atlanta: Centers for Disease Control and Prevention, National Center for Injury Prevention and Control; 2001.

22. Ministry of Community Development, Youth and Sports, Republic of Singapore. A National Council To Promote Water Safety. Media Release Apr 19, 2007. Available at: http://app.msf.gov.sg/Portals/0/Summary/pressroom/ WaterSafetyPressRelease.pdf. Accessed March 15, 2016.

23. National Water Safety Council (2007). National Water Safety Council Urges Employment of Lifeguards and Community Vigilance at All Private Swimming Pools [Media Release].

24. Toh KS, Tan C. Case update: swimming pools owners or operators may owe duty to provide properly trained lifeguards. In: Lee \& Lee [online]. Available at: http:// www.leenlee.com.sg/wp-content/uploads/2014/11/Case_Update_-_Swimming Pools_Owners_or_Operators_May_Owe_Duty_to_Provide_Properly_Trained_ Lifeguards-1.pdf. Accessed March 15, 2016

25. National Water Safety Council (27 May 2010). Launch of Singapore Standard (SS) 556: 2010 code of practice for the maintenance and design of aquatic facilities. [Media Release] Code of practice available at: http://www. singaporestandardseshop.sg. Accessed Mar 15, 2016.

26. Wallis BA, Watt K, Franklin RC, et al. Interventions associated with drowning prevention in children and adolescents: systematic literature review. Inj Prev 2015; 21:195-204

27. Morgenstern H, Bingham T, Reza A. Effects of pool-fencing ordinances and other factors on childhood drowning in Los Angeles County, 1990-1995. Am J Public Health 2000; 90:595-601.

28. Pearn J, Nixon J. Prevention of childhood drowning accidents. Med J Aust 1977; $1: 616-8$.

29. Fergusson DM, Horwood LJ. Risks of drowning in fenced and unfenced domestic swimming pools. N Z Med J 1984; 97:777-9.

30. Pitt WR, Balanda KP. Childhood drowning and near-drowning in Brisbane: the contribution of domestic pools. Med J Aust 1991; 154:661-5.

31. Centers for Disease Control and Prevention (CDC). Child drownings and near drownings associated with swimming pools--Maricopa County, Arizona, 1988 and 1989. MMWR Morb Mortal Wkly Rep 1990; 39:441-2.

32. Rimsza ME, Schackner RA, Bowen KA, Marshall W. Can child deaths be prevented? The Arizona Child Fatality Review Program experience. Pediatrics 2002; 110(1 Pt 1):e11.

33. Thompson DC, Rivara FP. Pool fencing for preventing drowning in children. Cochrane Database Syst Rev 2000; (2):CD001047.

34. Pearn JH, Wong RY, Brown J 3rd, et al. Drowning and near-drowning involving children: a five-year total population study from the city and county of Honolulu. Am J Public Health 1979; 69:450-4.

35. Milliner N, Pearn J, Guard R. Will fenced pools save lives? A 10-year study from Mulgrave Shire, Queensland. Med J Aust 1980; 2:510-1.

36. Quan L, Gore EJ, Wentz K, Allen J, Novack AH. Ten-year study of pediatric drownings and near-drownings in King County, Washington: lessons in injury 
prevention. Pediatrics 1989; 83:1035-40.

37. Logan P, Branche CM, Sacks JJ, Ryan G, Peddicord J. Childhood drownings and fencing of outdoor pools in the United States, 1994. Pediatrics 1998; 101:E3.

38. Morrison L, Chalmers DJ, Langley JD, Alsop JC, McBean C. Achieving compliance with pool fencing legislation in New Zealand: a survey of regulatory authorities. Inj Prev 1999; 5:114-8.

39. van Weerdenburg K, Mitchell R, Wallner F. Backyard swimming pool safety inspections: a comparison of management approaches and compliance levels in three local government areas in NSW. Health Promot J Austr 2006; $17: 37-42$. 